\title{
Bak Foong pills combined with metformin in the treatment of a polycystic ovarian syndrome rat model
}

\author{
WENHUI LIU ${ }^{1}$, WENPEI LIU ${ }^{2}$, YULING FU ${ }^{3}$, YAN WANG ${ }^{1}$ and YUANZHEN ZHANG ${ }^{1}$ \\ ${ }^{1}$ Center of Reproductive Medicine, Zhongnan Hospital of Wuhan University, Wuhan, Hubei 430071; \\ ${ }^{2}$ Translational Medicine Institute, Affiliated to The First People's Hospital of Chenzhou, University of South China, \\ Chenzhou, Hunan 423000; ${ }^{3}$ Department of Obstetrics and Gynecology, The Second Hospital of Yinzhou, \\ Ningbo, Zhejiang 315000, P.R. China
}

Received August 9, 2014; Accepted April 14, 2015

DOI: $10.3892 / 01.2015 .3466$

\begin{abstract}
The aim of the present study was to investigate the treatment effects and associated mechanism of Bak Foong pills (BFPs) combined with metformin in the treatment of polycystic ovarian syndrome (PCOS). BFPs and/or metformin were administrated to treat the PCOS rats, and the weights and morphologies of the ovary, uterus and adrenal gland were measured. The levels of fasting blood glucose (FBG), serum testosterone ( $\mathrm{T})$, luteinizing hormone, fasting insulin (FIN) and insulin-like grow th factor-1 were also measured, and the homeostasis model assessment of insulin resistance (HOMA-IR) was calculated. The expression level of androgen receptor (AR) in the ovarian tissue, and the cytochrome $\mathrm{P} 450$ cholesterol side-chain cleavage enzyme (P450scc) mRNA levels in the ovary and adrenal tissues were detected. The levels of T, FIN, FBG and HOMA-IR in the combination group were significantly reduced; the wet weights of the ovary and the adrenal gland were decreased significantly, while that of the uterus was increased, and the histological morphology benignly recovered. The rats of each treatment group all experienced restored ovulation. The AR expression level in the treatment group was reduced, and the P450scc mRNA levels in the ovary and the adrenal gland of the combined treatment group were decreased. BFPs combined with metformin significantly affected PCOS, and the possible mechanism involved in the treatment may have been through the reduction of P450scc generation. BFPs may reduce the androgen levels, thus allowing the ovary to restore ovulation.
\end{abstract}

Correspondence to: Professor Yuanzhen Zhang, Center of Reproductive Medicine, Zhongnan Hospital of Wuhan University, 169 East Lake Road, Wuhan, Hubei 430071, P.R. China

E-mail: yuanzhenzhang@126.com

Key words: polycystic ovarian syndrome, Bak Foong pills, metformin, cytochrome $\mathrm{P} 450$ cholesterol side-chain cleavage enzyme, insulin-like factor-1

\section{Introduction}

Polycystic ovarian syndrome (PCOS) is a female endocrine system disorder disease that affects the reproductive function of $9-18 \%$ of women of childbearing age (1). Over the years, a number of scholars have studied PCOS from a range of aspects, including its biochemistry, and endocrine and ovarian morphology. The characteristic clinical manifestation of this disease is its high heterogeneity, including high androgen, insulin resistance (IR) and glucose metabolism levels, thus the diagnosis and treatment remains difficult. PCOS at the childbearing age is the continuation of adolescent PCOS, which can increase the risks of cardiovascular system and glucose metabolism abnormalities in old age, as well as resulting in disorders of psychological and social cognition functions (2-7). Therefore, the effective treatment of adolescent PCOS is of particular importance (8). However, the effectiveness of pure Western or traditional Chinese medicine (TCM) treatment in adolescent PCOS has not been satisfactory (9-14).

Bak Foong pills (BFP) are a traditional Chinese gynecological medicine that is believed to combine the bioactivities of 'warm-reinforcing', nourishing the Yin, resolving blood stasis and harmonizing the body, and are widely used in the prevention and treatment of obstetric and gynecological diseases. The insulin sensitizer, metformin, can cause reductions in body weight, restore ovulation, increase the rate of pregnancy and reduce the number of pregnancy complications (15-18). Our previous study successfully established a PCOS rat model that could simulate the clinical and biochemical characteristics of PCOS. The present study aimed to use combined BFP and metformin to treat the adolescent PCOS animal model, aiming to investigate the advantages of the joint application of Chinese and Western medicines in the treatment of adolescent PCOS.

\section{Materials and methods}

Animals. A total of 50 healthy, female, 21-day-old Sprague Dawley rats (specific pathogen free level), each weighing 40-50 g, were provided by the Experimental Animal Center, School of Medicine of Wuhan University (Wuhan, China). The rats were housed on a 12/12 h light/dark cycle, in a 
temperature-controlled $\left(20-26^{\circ} \mathrm{C}\right)$ and humidity-controlled (50-70\%) room, with food and water available ad libitum, except when food and water were withdrawn for $10 \mathrm{~h}$ prior to sampling. The present study was performed in strict accordance with the recommendations in the Guide for the Care and Use of Laboratory Animals of the National Institutes of Health (19). The animal use protocol was reviewed and approved by the Institutional Animal Care and Use Committee of Zhongnan Hospital of Wuhan University.

Establishment of a PCOS model and grouping. The Sprague Dawley rats were established as a PCOS model from 23 days old through use of DHEA powder (99.72\%; Hubei Fangtong Pharmaceutical Co., Ltd., Huangshi, China) and soybean oil injections (Tieling Beiya Pharmaceutical Oil Co., Tieling, China), as described previously (20).

Following this, the animal models were randomly divided into four groups according to the random number table method as follows: The model control group (group B; $2 \mathrm{ml}$ solution of $0.9 \% \mathrm{NaCl}$ daily, intragastric), the BFP treatment group (group C; $2 \mathrm{ml}$ solution including $0.9 \% \mathrm{NaCl}$ and $3.6 \mathrm{~g} / \mathrm{kg} / \mathrm{day}$ of BFP, intragastric), the metformin treatment group (group D; $2 \mathrm{ml}$ solution including $0.9 \% \mathrm{NaCl}$ and $300 \mathrm{mg} / \mathrm{kg} / \mathrm{day}$ of metformin, intragastric) and the combination treatment group (group E; administered with BFP and metformin as described for groups $\mathrm{C}$ and $\mathrm{D}$ ). A solvent control group (group A), in which the animals received $0.9 \% \mathrm{NaCl}$ solution only, was also created; no DHEA, BFP or metformin treatments were administered to this group. A total of 10 rats were allocated to each group, with each group administered different medication procedures using BFP (Beijing Tongrentang Pharmaceutical Factory, Beijing, China) and metformin hydrochloride enteric-coated tablets (Guizhou Shengjitang Pharmaceutical Co., Ltd., Guiyang, China). A daily vaginal smear was obtained to monitor the ovulation of each group, and after 3 consecutive weeks of intragastric drug administration, the rats were fasted for $12 \mathrm{~h}$ prior to sacrifice by an overdose of anaesthesia via intraperitoneal injection. Venous blood was obtained to measure the fasting blood glucose (FBG) level, and the ovaries, uterus and adrenal glands were removed to be weighed. One ovary and adrenal gland were placed in $10 \%$ formalin solution for fixation, while the others were quickly frozen in liquid nitrogen and stored at $-70^{\circ} \mathrm{C}$ for future tests.

Changes to the estrous cycle. Hematoxylin-eosin (HE) staining was performed on vaginal smears taken consecutively for 21 days, and the morphological changes of shed vaginal cells were observed, as previously described $(21,22)$. Each typical estrous cycle, marked by the observation of ovulation (normally every 5-6 days in rats), was assigned a score of 3 .

Organ weight. The wet weights of the uterus, one ovary and one adrenal gland were recorded.

$F B G$. An FBG meter was used to test FBG levels after $12 \mathrm{~h}$ of fasting.

Radioimmunoassay. The serum luteinizing hormone (LH), testosterone (T), fasting insulin (FIN) and insulin-like growth factor-1 (IGF-1) levels were detected by radioimmunoassay using T, IGF-1 and LH radioimmunoassay kits (Tianjin Jiuding Medical Biological Engineering Co., Ltd., Tianjin, China) and an insulin radioimmunoassay kit (Beijing Atom High-Tech Jinhui Radiation Technology Application Co., Ltd., Beijing, China), following the manufacturer's instructions.

Morphology. The general anatomical and HE morphological characteristics of the ovary, uterus and adrenal gland were analyzed using $10 \%$ formalin fixation, paraffin-embedding and conventional sectioning, followed by HE staining and microscopic examination.

Immunohistochemistry. Immunohistochemistry was performed using the AR primary antibody (BIOSS, Beijing, China), slide-sticking reagent Poly-lysine, AB staining kit (ED1022) and biotinylated goat anti-mouse immunoglobulin G (Wuhan Boster Biological Technology, Ltd., Wuhan, China). The immunohistochemistry was performed to analyze the AR expression of each group by calculating the percentage of positive cells. Five fields of vision were selected for each slice and analyzed under a high-power optical microscope (BX41-12J02, Olympus Corporation, Tokyo, Japan; magnification, $\mathrm{x} 400$ ), and 500 cells were counted to calculate the positive cell percentage.

P450scc mRNA expression in the ovary and adrenal tissues. The semi-quantitative reverse transcription (RT)-polymerase chain reaction (PCR) method was used for the analysis of P450scc mRNA expression in the ovary and adrenal tissues using a PTC-200 PCR instrument (Bio-Rad Laboratories, Inc., Hercules, CA, USA). The total RNA was extracted from the ovary and adrenal tissues $\left(\sim 0.1-0.5 \mathrm{~cm}^{3}\right)$ with TRIzol reagent (Invitrogen Life Technologies, Carlsbad, CA, USA) according to the manufacturer's instructions. The mRNA was dissolved in double distilled water and the absorbance $\left(\mathrm{A}_{260} / \mathrm{A}_{280}\right)$ was measured by an ultraviolet (UV) spectrophotometer (UV-2000; Shanghai Precision Instrument Co.,Ltd., Shanghai, China) to calculate the concentration.

The reverse synthesis of first-strand complementary (c) DNA was performed using a kit purchased from Thermo Fisher Scientific (Vilnius, Lithuania), following the manufacturer's instructions; the reaction volume was $20 \mu \mathrm{l}$, containing $4 \mu \mathrm{l}$ of $5 \mathrm{X}$ RT buffer, $2 \mu \mathrm{l}$ of $10 \mathrm{mM}$ dNTP mixture, $1 \mu \mathrm{l}$ of $10 \mathrm{pmol} / \mu \mathrm{l}$ oligo (dT), $1 \mu \mathrm{l}$ of $10 \mathrm{U} / \mu 1$ RNase inhibitor, $1 \mu 1$ of avian myeloblastosis virus reverse transcriptase $(5 \mathrm{U} / \mu \mathrm{l})$, $2 \mu \mathrm{l}(1 \mu \mathrm{g})$ of RNA template, and $9 \mu \mathrm{l}$ of RNase-free $\mathrm{H}_{2} \mathrm{O}$. The RT conditions were $50^{\circ} \mathrm{C}$ for $45 \mathrm{~min}, 95^{\circ} \mathrm{C}$ for $5 \mathrm{~min}$ and ice-bath for $5 \mathrm{~min}$. PCR consisted of 35 cycles of $94.0^{\circ} \mathrm{C}$ for $2 \mathrm{~min}, 94.0^{\circ} \mathrm{C}$ for $30 \mathrm{~s}, 57.8^{\circ} \mathrm{C}$ for $30 \mathrm{~s}$ and $72.0^{\circ} \mathrm{C}$ for $30 \mathrm{~s}$, followed by extension at $72.0^{\circ} \mathrm{C}$ for $5 \mathrm{~min}$ The primers were designed by Primer Premier software (version 5.0), and synthesized by China Shanghai Biological Engineering Co. (Shanghai, China), and the sequences were as follows: P450scc upstream primer, 5'-ATGCCTCCAGACTTATTTCG-3' and downstream primer, 5'-AAGCACCAGTCGTTCACA-3', with a 462-bp amplified fragment length; and GAPDH upstream primer, 5'-ATTGTCAGCAATGCATCCTG-3' and downstream primer, 5'-GTAGGCCATGAGGTCCACCA-3', with a 568-bp amplified fragment length. 
For the detection and semi-quantitation of the RT-PCR products, $2 \%$ agarose gel electrophoresis was used, and after ethidium bromide staining, the electrophoresis result was observed under UV light. The Bio-Rad Doc Gel 2000 (Bio-Rad Laboratories, Inc.) gel imaging system was used to quantify the P450scc mRNA expression and the exponentially amplified GAPDH bands, respectively, by generating a transmittance value; the ratio of P450scc mRNA and GAPDH was used to reflect the content of P450scc mRNA in the ovary and adrenal tissues.

Statistical analysis. The data were expressed as the mean \pm standard deviation, and the SPSS 15.0 statistical software (SPSS, Inc., Chicago, IL, USA) was used for the statistical analysis. The intergroup pairwise comparison used a one-way analysis of variance. $\mathrm{P}<0.05$ was considered to indicate a statistically significant difference.

\section{Results}

Changes of estrous cycle. At 2 to 3 days after the withdrawal of dehydroepiandrosterone from the adrenal glands, the vaginal exfoliated cells of groups B, C, D and E transformed from sustained keratinocytes in the modeling phase to a large number of white blood cells in the estrous phase. With each different medication, the estrous cycles of the groups exhibited a variety of changes. Each typical estrous cycle (demonstrated by ovulation) was recorded with a score of 3 points, and consecutive vaginal smears for 21 days revealed that group A exhibited regular estrous cycles, which differed significantly from group $\mathrm{B}(\mathrm{P}=0.002$; Table I). The comparison between group $\mathrm{A}$ and groups $\mathrm{C}$ and $\mathrm{D}$ also revealed significant differences $(\mathrm{P}=0.037$ and $\mathrm{P}=0.013$, respectively), suggesting that the recovery of the estrous cycle in groups $\mathrm{C}$ and D was poor. Compared with groups B, C and D, group E exhibited a significant difference, while no significant difference was observed when compared with group $\mathrm{A}(\mathrm{P}=0.136)$, indicating that the recovery level of the estrous cycle in group E was close to the solvent control group, with 3-4 estrous cycles observed (Table I). These results suggested that combined metformin and BFP had a significantly greater effect with regard to the recovery of ovulation compared with BFP or metformin alone.

Wet weights of the ovary, uterus and adrenal gland. Compared with those of group B, the wet weights of the ovary and adrenal gland of group A were significantly reduced ( $\mathrm{P}=0.032$ and $\mathrm{P}=0.022$, respectively), while those of the uterus were increased $(\mathrm{P}=0.041)$. Compared with group $\mathrm{A}$, the wet weights of ovary, uterus and adrenal gland of the group $\mathrm{D}$ were significantly increased $(\mathrm{P}=0.27)$, decreased $(\mathrm{P}=0.038)$ and increased $(\mathrm{P}=0.042)$, respectively. The wet weights of the ovary, uterus and adrenal gland of groups $\mathrm{A}$ and $\mathrm{E}$ exhibited no significant differences $(\mathrm{P}=0.132, \mathrm{P}=0.211$ and $\mathrm{P}=0.082$, respectively; Table II).

General morphological and HE section observations. The ovary in group A was ruddy, with multiple lutea on the ovarian surface. In group B, the ovarian surface was pale, the volume of the ovary was increased and multiple dilated
Table I. Estrous cycle scores for each group.

\begin{tabular}{lcr}
\hline Group & $\mathrm{n}$ & \multicolumn{1}{c}{ Score } \\
\hline A & 10 & $14.41 \pm 1.27$ \\
B & 10 & $2.72 \pm 2.63^{\mathrm{a}}$ \\
C & 10 & $6.01 \pm 2.83^{\mathrm{a}}$ \\
D & 10 & $4.53 \pm 1.58^{\mathrm{a}}$ \\
E & 10 & $9.98 \pm 3.68^{\mathrm{b}}$
\end{tabular}

${ }^{\mathrm{a}} \mathrm{P}<0.05$ vs. group $\mathrm{A} ;{ }^{\mathrm{b}} \mathrm{P}>0.05$ vs. group $\mathrm{A}$. Data are presented as the mean \pm standard deviation.

transparent vesicles were present on the ovarian surface. Under the microscope, group A exhibited multiple lutea and ovarian follicles at the different developmental stages. The anatomical structures of the follicular theca cell layers, granule cell layers and eggs at all levels were clear. In group B, more vacuoles, oocytes or the corona radiata had disappeared, and the number of lutea was markedly reduced. The general morphological observation towards the treatment group revealed that the $4 / 10$ rats in group C, 5/10 in group D and $1 / 10$ in group $\mathrm{E}$ exhibited multiple thin transparent small follicles on the ovarian surface. Endoscopy revealed that the lutea and normal follicles of each group were increased in number by varying degrees, among which, the observation indices of group E were similar to those of group A.

With regard to the uterus, the gross morphology exhibited no significant differences among the groups. In groups B and $\mathrm{D}$, the size of the peri-uterus fat pad was increased and the uterine length was decreased compared with the other groups. Under the endoscope, the endometrial thickness of groups B and D were significantly thickened compared with groups $\mathrm{A}$ and $\mathrm{E}$. While there was no significant difference among the remaining groups.

With regard to the adrenal gland, no significant difference existed in the gross morphology among the groups. Under the microscope, the zona reticularis of group B was markedly enlarged compared with the control group, and was irregularly stretched into the zona fasciculata, with rich capillaries. In groups A and E, the adrenal cortical structure was clear and structured. Groups $\mathrm{C}$ and $\mathrm{D}$ exhibited a benign recovery trend in comparison to group B.

$F B G, F I N$ and the homeostasis model assessment of IR (HOMA-IR). Compared with group B, the level of FBG, FIN and HOMA-IR differed significantly in groups A, C, D and $\mathrm{E}$; the IR of groups C, D and E was improved. Compared with the group $\mathrm{C}$, the level of FBG, FIN and HOMA-IR in groups A, D and E exhibited a significant difference, while the differences among groups A, D and E were not significant (Table III).

T, LH and IGF-1 levels. With regard to T levels, the levels in groups $\mathrm{A}, \mathrm{C}, \mathrm{D}$ and $\mathrm{E}$ were significantly lower than those in group $\mathrm{B}(\mathrm{P}=0.031, \mathrm{P}=0.040, \mathrm{P}=0.022$ and $\mathrm{P}=0.034$, respectively. With regard to the LH levels, the levels in groups $\mathrm{B}$ and D were marginally higher than those in groups $\mathrm{A}, \mathrm{C}$ 
Table II. Wet weights of ovary, uterus and adrenal gland in the different treatment groups (mean \pm standard deviation).

\begin{tabular}{llccc}
\hline & & \multicolumn{3}{c}{ Wet weight, $\mathrm{g}$} \\
\cline { 3 - 4 } Group & $\mathrm{n}$ & Ovary & Uterus & Adrenal gland \\
\hline $\mathrm{A}$ & 10 & $0.0412 \pm 0.0039$ & $0.4872 \pm 0.0164$ & $0.0346 \pm 0.0035$ \\
$\mathrm{~B}$ & 10 & $0.0537 \pm 0.0025^{\mathrm{a}}$ & $0.3608 \pm 0.0673^{\mathrm{a}}$ & $0.0396 \pm 0.0021^{\mathrm{a}}$ \\
$\mathrm{C}$ & 10 & $0.0474 \pm 0.0024$ & $0.4753 \pm 0.0532$ & $0.0340 \pm 0.0037$ \\
$\mathrm{D}$ & 10 & $0.0528 \pm 0.0036^{\mathrm{a}}$ & $0.3452 \pm 0.0872^{\mathrm{a}}$ & $0.0374 \pm 0.0065^{\mathrm{a}}$ \\
$\mathrm{E}$ & 10 & $0.0424 \pm 0.0023^{\mathrm{b}}$ & $0.4921 \pm 0.0542^{\mathrm{b}}$ & $0.0352 \pm 0.0045^{\mathrm{b}}$ \\
\hline
\end{tabular}

${ }^{\mathrm{a}} \mathrm{P}<0.05$ vs. group $\mathrm{A} ;{ }^{\mathrm{b}} \mathrm{P}>0.05$ vs. group $\mathrm{A}$. Data are presented as the mean \pm standard deviation.

Table III. FBG, FIN and HOMA-IR of the experimental groups.

\begin{tabular}{lcrrl}
\hline Group & $\mathrm{n}$ & FIN, mIU/l & FBG, mmol/l & HOMA-IR \\
\hline A & 10 & $10.50 \pm 1.41$ & $6.59 \pm 0.59$ & $3.08 \pm 0.58^{\mathrm{a}}$ \\
B & 10 & $12.62 \pm 2.14$ & $10.73 \pm 0.87$ & $7.45 \pm 1.22$ \\
C & 10 & $10.85 \pm 1.76$ & $7.44 \pm 0.92$ & $3.61 \pm 0.88^{\mathrm{a}}$ \\
D & 10 & $9.53 \pm 0.81$ & $6.37 \pm 0.78$ & $2.71 \pm 0.46^{\mathrm{a}, \mathrm{b}}$ \\
E & 10 & $9.76 \pm 1.78$ & $6.93 \pm 0.76$ & $3.02 \pm 0.69^{\mathrm{a}, \mathrm{b}}$ \\
\hline
\end{tabular}

${ }^{\mathrm{a}} \mathrm{P}<0.05$ vs. group $\mathrm{B}$; and ${ }^{\mathrm{b}} \mathrm{P}>0.05$ vs. group $\mathrm{A}$. FBG, fasting blood glucose; FIN, fasting insulin; HOMA-IR, homeostasis model assessment of insulin resistance. Data are presented as the mean \pm standard deviation

Table IV. T, LH and IGF-1 levels of the experimental groups.

\begin{tabular}{lcccc}
\hline Group & $\mathrm{n}$ & $\mathrm{T}, \mathrm{ng} / \mathrm{ml}$ & $\mathrm{LH}, \mathrm{mIU} / \mathrm{ml}$ & \multicolumn{1}{c}{ IGF-1 } \\
\hline A & 10 & $4.49 \pm 0.79^{\mathrm{a}}$ & $8.80 \pm 0.82$ & $78.18 \pm 9.66$ \\
B & 10 & $6.51 \pm 1.25$ & $9.13 \pm 1.15$ & $53.68 \pm 11.09$ \\
C & 10 & $4.35 \pm 1.43^{\mathrm{a}}$ & $8.49 \pm 0.86$ & $79.79 \pm 11.84$ \\
D & 10 & $4.54 \pm 1.23^{\mathrm{a}}$ & $9.02 \pm 1.24$ & $51.01 \pm 11.50$ \\
E & 10 & $4.01 \pm 1.69^{\mathrm{a}}$ & $8.89 \pm 0.89$ & $69.18 \pm 9.78$
\end{tabular}

${ }^{\mathrm{a}} \mathrm{P}<0.05$ vs. group B. T, testosterone; $\mathrm{LH}$, luteinizing hormone; IGF-1, insulin-like growth factor-1. Data are presented as the mean \pm standard deviation.

and $\mathrm{E}$, and the intergroup difference was not significant. With regard to the IGF-1 levels, the levels in groups A and C were slightly higher than those in groups B, D and E, and the intergroup difference was not significant (Table IV).

Ovarian AR expression. The cytoplasm and nuclei of the theca cells of the control group and each treatment group all exhibited AR expression, with brown-stained cytoplasm and nuclei representing positive staining. The comparison between groups $\mathrm{B}$ and $\mathrm{D}$ and groups $\mathrm{A}$ and $\mathrm{E}$ revealed no significant difference, whereas the positive expression rates in groups B and D were higher than those in groups A and E, and this difference was statistically significant.
Relative expression of P450scc mRNA (P450scc/GAPDH). The ovarian and uterine P450scc mRNA levels in group A were significantly reduced compared with the levels in group $\mathrm{B}(\mathrm{P}=0.043$ and $\mathrm{P}=0.031$, respectively). Compared with group $\mathrm{B}$, the ovarian $\mathrm{P} 450 \mathrm{scc}$ mRNA content of group D was not significantly different $(\mathrm{P}=0.12)$, while that in the adrenal tissue was significantly reduced compared with group $\mathrm{B}$ $(\mathrm{P}=0.042)$. The comparison of ovarian and uterine $\mathrm{P} 450 \mathrm{scc}$ mRNA contents in groups $\mathrm{A}$ and $\mathrm{E}$ exhibited no statistically significant difference $(\mathrm{P}=0.126$ and $\mathrm{P}=0.229$, respectively; Table V), which may indicate a level of hormone recovery in group E.

\section{Discussion}

The purpose of PCOS treatment in Chinese and Western medicine are the same, and mainly include the following aspects (23): i) To realize a normal weight; ii) to reduce the androgen levels; iii) to induce ovulation in patient's who desire fertility; iv) to reduce the risks of cardiovascular diseases; v) to avoid the serious consequences caused by hyperinsulinemia; and vi) to protect the endometrium.

The current PCOS treatment methods in Western medicine can mainly be divided into three categories according to a patient's age and fertility requirements. For adolescent PCOS patients, menoxenia is the chief complaint, thus conditioning the menstrual cycle and reducing the androgen levels would be the main treatment purposes. Women of reproductive age mainly exhibit infertility as the chief complaint, thus, the treatment should mainly focus on the promotion of ovulation and reproductive assistance. When PCOS patients enter middle-age and old-age, their condition is normally accompanied by hypertension, hyperlipidemia and diabetes. The general medical treatment for all internal-medicine PCOS patients should consist of the basics of weight control, diet structure changes and enhancement of physical activity (24). The treatment program should be symptomatic supportive treatment, with a core focus on reducing the androgen level. Adolescent PCOS would affect the adolescent psychology and physiological development, thus the appropriate treatment and life guidance would aid in the subsequent adult health state $(25,26)$.

P450scc is the steroid hormone-synthesized key enzyme and rate-limiting enzyme encoded by the CYP11a gene. Clinical genetics studies have considered that CYP11a is the 
Table V. P450scc/GAPDH mRNA values of the experimental groups.

\begin{tabular}{lccc}
\hline Group & $\mathrm{n}$ & Ovary & Adrenal gland \\
\hline A & 10 & $0.488705 \pm 0.189914^{\mathrm{a}}$ & $0.379609 \pm 0.089713^{\mathrm{a}}$ \\
B & 10 & $0.639585 \pm 0.096282$ & $0.539183 \pm 0.076491$ \\
C & 10 & $0.514505 \pm 0.026796$ & $0.433797 \pm 0.085209$ \\
D & 10 & $0.629585 \pm 0.037362$ & $0.539585 \pm 0.038914$ \\
E & 10 & $0.507625 \pm 0.092842^{\mathrm{b}}$ & $0.398756 \pm 0.073661^{\mathrm{b}}$
\end{tabular}

${ }^{\mathrm{a}} \mathrm{P}<0.05$ vs. group $\mathrm{B} ;{ }^{\mathrm{b}} \mathrm{P}>0.05$ vs. group A. Data are presented as the mean \pm standard deviation.

susceptibility gene of PCOS, which promotes the synthesis and secretion of androgens. In the present study, the results of RT-PCR analysis revealed that the ovarian P450scc mRNA content in the PCOS patients was higher than that in the adrenal gland. The P450scc mRNA level in the combined medication group was significantly lower than the PCOS model blank group, and the difference was significant, consistent with the results found by Li et al (27). However, compared with the solvent control group, the difference was not statistically significant. In the present study, the ovarian AR expression level in the combination group was reduced compared with the PCOS model blank group, and the difference was significant. There was, however, no statistical significance when compared with the solvent control group. The comparison between the metformin group and the model control group showed no statistically significant difference, while compared with the BFP group, the AR expression was significantly higher. After combining the situations of all groups, the analysis revealed that the recovery situations of ovulation function were closely associated with the ovarian microenvironment; simply reducing the serum insulin level may reduce the serum androgen level, but the local ovarian androgen level could not be improved so the recovery of ovulation function would be poor. These results further confirmed that the recovery of ovarian function required changes to the basic ovarian environment to make it balanced and in harmony. In the present study, the combination therapy exhibited synergistic effects; the combined application of BFP and metformin was able to reduce the $\mathrm{P} 450 \mathrm{scc}$ production in the local ovary, possibly through regulating the multiple target organs, such as the gonadal axis and the adrenal gland, while it had no effect towards the $\mathrm{LH}$ and exhibited a role in reducing the levels of AR receptor. Thus, it could be presumed that it could basically reduce the androgen levels and biological activities, thus promoting follicular development and improving the ovulation dysfunction.

The Chinese pharmaceutical company Beijing Tongrentang uses pure breed black-bone chickens as the raw materials for BFP, combined with 19 other medical herbs that nourish the Yin and reinforce the kidney (28-30). The prescription has the bioactivities of enriching and coagulating the blood, regulating lipids, relieving pain, estrogen or androgen-like effects, corticotropin-like effects and anti-fatigue effects. Certain constituents, such as Deer's antlers and ootheca mantidis, are believed to reinforce the liver and kidney, benefit the blood and essence, benefit the kidney and enrich the Yang; the prepared rhizome of Rehmannia glutinosa, Angelica sinensis and Paeonia lactiflora could nourish the blood and promote blood circulation; Rhizoma chuanxiong and Salvia miltiorrhiza could also promote the blood circulation; ginseng, Astragalus membranaceus, rhizoma dioscoreae and dried Euryale ferox could strengthen the spleen and profit the Qi, dissipate dampness and stop leukorrhea; concha ostreae (oyster shell), Rehmannia glutinosa, radix asparagi, radix stellariae and herba Artemisiae annuae, could nourish the Yin and digest phlegm; Rhizoma Cyperi could reorganize the liver Qi; and Glycyrrhiza uralensis could reconcile the other drugs. The herbs were reasonably combined, and this prescription may adjust the dysfunction of the 'kidney-Tiangui-Chongren-uterus' axis from a whole aspect (26), thus reducing the androgen level, promoting the follicular development and improving the ovulation disorders. The therapeutic mechanisms may include three aspects: Firstly, the TCM acts on the ovary, regulating the functions of the hypothalamus-pituitary-ovarian axis; secondly, it acts on the adrenal gland, regulating the hypothalamus-pituitary-adrenal gland axis; and lastly, its active ingredient, ligustrazine, acts on the uterus, regulating the endometrial fluctuation rhythm, aiding in the recovery of menstruation and embryo implantation $(31,32)$.

The excessive level of secreted growth hormone in adolescence may cause the IR. The IR is closely associated with the hyperandrogen, which could not only stimulate the ovary to produce more androgen, but also reduce the binding between the sex hormone and protein, therefore, the insulin sensitizer is considered to have great potential for PCOS treatment. Morciano et al (33) performed a retrospective analysis and found that obese or non-obese PCOS women, who had undergone an oral glucose tolerance test and had a recorded IR index, all exhibited the metabolic disorder of IR. Thus, the study recommended that all PCOS women should be examined for IR. Stepto et al (34) found that body mass index-associated IR existed inside the bodies of PCOS patients, and that early treatment could prevent concurrent diabetes in high-risk populations. This study also found that PCOS patient's exhibited increasing HOMA-IR and IR, and that an insulin sensitizer could ameliorate this metabolic disorder. The PCOS patients that develop IR in the adolescence should be administered insulin sensitizers as early as possible, so that the metabolism can be effectively regulated, and cardiovascular disease and diabetes can be prevented (35); this finding was in accordance with the present study. 
The results of the present study suggested that BFP, metformin tablets and the combination of these two drugs had a positive effect in the treatment of adolescent PCOS. The combination group exhibited much greater effects than the pure metformin or BFP groups, and the efficacy evaluation revealed that the difference was significant compared with the model control group. While the difference was not statistically significant when compared with the solvent control group, the combination group exhibited reduced $\mathrm{T}$, FBG, FIN and HOMA-IR levels compared than with the model control group, and the differences were significant. The comparison between the combination group and the model control group revealed that the gross morphologies and pathologies of the ovary, uterus and adrenal gland exhibited benign recovery changes; among these cases, the ovarian wet weight was reduced, with significantly increased luteum generation on the surface, while only $1 / 10$ cystic dilated vesicles were observed. The estrous cycle of the combination group recovered well, suggesting the existence of regular ovulation, while the model control group lost the regular estrous cycle, suggesting no ovulation or that ovulation was rare. The indices of the pure BFP or metformin groups all exhibited a positive recovery trend, and exhibited no significant difference when compared with the model control group. The simple BFP treatment improved the estrous cycle, but was not markedly effective in reducing the IR, while metformin exhibited a significant effect in improving the IR, which effectively reduced FIN and HOMA-IR; there was no difference with the model group with regard to the recovery of the estrous cycle. Therefore, the combined application of metformin and BFP exhibited a synergistic effect towards the PCOS treatment, possibly improving the IR and fundamentally complementing the renal fluids and 'Tiangui', regulating the gonadal axis, promoting follicular development, activating the blood circulation and dissipating congestion, as well as promoting ovulation.

The combined application of Chinese and Western medicine could reduce the side-effects of Western medicine, and make up for the disadvantages of pure Chinese medicine treatment, namely the slow effect and long treatment course. The efficacy of combination therapy was better than that of pure TCM or Western medicine. Although the present study was an animal experiment, it was still a meaningful attempt to combine Chinese and Western medicine to treat PCOS, thus, it could provide a theoretical basis for the further study of combination therapy for PCOS treatment.

\section{Acknowledgements}

This study was supported by Hubei Provincial Health Department (project number JBX3022).

\section{References}

1. March WA, Moore VM, Willson KJ, Phillips DI, Norman RJ and Davies MJ: The prevalence of polycystic ovary syndrome in a community sample assessed under contrasting diagnostic criteria. Hum Reprod 25: 544-551, 2010.

2. Nasiri Amiri F, Ramezani Tehrani F, Simbar M, Montazeri A and Mohammadpour Thamtan RA: The experience of women affected by polycystic ovary syndrome: A qualitative study from Iran. Int J Endocrinol Metab 12: e13612, 2014.
3. Hung JH, Hu LY, Tsai SJ, et al: Risk of psychiatric disorders following polycystic ovary syndrome: A nationwide population-based cohort study. PLoS One 9: e97041, 2014.

4. Ezeh U, Pall M, Mathur R and Azziz R: Association of fat to lean mass ratio with metabolic dysfunction in women with polycystic ovary syndrome. Hum Reprod 29: 1508-1517, May 9, 2014 (Epub ahead of print).

5. Diamanti-Kandarakis E, Spritzer PM, Sir-Petermann T and Motta AB: Insulin resistance and polycystic ovary syndrome through life. Curr Parm Des 18: 5569-5576, 2012.

6. Wiltgen D and Spritzer PM: Variation in metabolic and cardiovascular risk in women with different polycystic ovary syndrome phenotypes. Fertil Steril 94: 2493-2496, 2010.

7. Di Domenico K, Wiltgen D, Nickel FJ, Magalhães JA, Moraes RS and Spritzer PM: Cardiac autonomic modulation in polycystic ovary syndrome: Does the phenotype matter? Fertil Steril 99: 286-292, 2013.

8. Pinola P, Lashen H, Bloigu A, et al: Menstrual disorders in adolescence: A marker for hyperandrogenaemia and increased metabolic risks in later life? Finnish general populationbased birth cohort study. Hum Reprod 27: 3279-3286, 2012.

9. Legro RS: The current status for metformin use in reproductive medicine. In: Reproductive Endocrinology and Infertility. Springer, New York, pp183-pp189, 2010.

10. Lai L, Flower A, Moore M and Lewith G: Chinese herbal medicine and polycystic ovary syndrome: A randomized feasibility and pilot study in the United kingdom. J Altern Complement Med 20: A61-A62, 2014.

11. Lai L, Li X, Flower A, et al: Chinese herbal medicine for oligomenorrhoea and amenorrhoea in polycystic ovary syndrome: A systematic review and meta-analysis. J Altern Complement Med 20: A129, 2014.

12. Ma RJ, Zhou J, Fang JQ, Yang DH and Qu F: Combination of acupuncture and chinese medicinal herbs in treating model rats with polycystic ovary syndrome. Afr J Tradit Complement Altern Med 8: 353-361, 2011.

13. Zhang J, Li T, Zhou L, et al: Chinese herbal medicine for subfertile women with polycystic ovarian syndrome. Cochrane Database Syst Rev 8: CD007535, 2010.

14. Raja-Khan N, Stener-Victorin E, Wu X and Legro RS: The physiological basis of complementary and alternative medicines for polycystic ovary syndrome. Am J Physiol Endocrinol Metab 301: E1-E10, 2011.

15. Bargiota A and Diamanti-Kandarakis E: The effects of old, new and emerging medicine on metabolic aberration in PCOS. Ther Adv Endocrinol Metab 3: 27-47, 2012.

16. Ladson G, Dodson WC, Sweet SD, et al: The effects of metformin with lifestyle therapy in polycystic ovary syndrome: A randomized double-blind study. Fertil Steril 95: 1059-1066, 2011.

17. Diamanti-Kandarakis E, Christakou CD, Kandaraki E and Economou FN: Metformin: An old medication of new fashion: Evolving new molecular mechanisms and clinical implications in polycystic ovary syndrome. Eur J Endocrinol 162: 193-212, 2010.

18. Palomba S, Falbo A, Russo T, Orio F, Tolino A and Zullo F: Systemic and local effects of metformin administration in patients with polycystic ovary syndrome (PCOS): Relationship to the ovulatory response. Hum Reprod 25: 1005-1013, 2010.

19. Committee for the Update of the Guide for the Care and Use of Laboratory Animals; Institute for Laboratory Animal Research; Division on Earth and Life Studies; National Research Council: Guide For The Care And Use Of Laboratory Animals. 8th edition. National Academies Press, Washington, D.C., 2011.

20. Singh KB: Persistent estrus rat models of polycystic ovary disease: An update. Fertil Steril 84 (Suppl 2): 1228-1234, 2005.

21. Goldman JM, Murr AS and Cooper RL: The rodent estrous cycle: Characterization of vaginal cytology and its utility in toxicological studies. Birth Defects Res B Dev Reprod Toxicol. 80, 84-97, 2007.

22. Cora MC, Kooistra L and Travlos G: Vaginal Cytology of the Laboratory Rat and Mouse: Review and Criteria for the Staging of the Estrous Cycle Using Stained Vaginal Smears. Toxicol Pathol: Mar 3, 2015 (Epub ahead of print). doi: $10.1177 / 0192623315570339$.

23. Sharma ST and Nestler JE: Prevention of diabetes and cardiovascular disease in women with PCOS: Treatment with insulin sensitizers. Best Pract Res Clin Endocrinol Metab 20: 245-260, 2006. 
24. Smith JW and Taylor JS: Polycystic ovary syndrome: Evidence-based strategies for managing symptoms and preventing long-term sequelae. Nurs Womens Health 15: 402-410, 2011.

25. Mani H, Potdar N and Gleeson H: How to manage an adolescent girl presenting with features of polycystic ovary syndrome (PCOS); an exemplar for adolescent health care in endocrinology. Clin Endocrinol (Oxf) 81: 652-656, 2014.

26. Auble B, Elder D, Gross A and Hillman JB: Differences in the management of adolescents with polycystic ovary syndrome across pediatric specialties. J Pediatr Adolesc Gynecol 26: 234-238, 2013.

27. Li H, Chen Y, Yan LY and Qiao J: Increased expression of P450scc and CYP17 in development of endogenous hyperandrogenism in a rat model of PCOS. Endocrine 43: 184-190, 2013.

28. Zheng XY, Peng Y, Ren DQ, et al; State Pharmacopoeia Commission of the People's Republic of China: Pharmacopoeia of the People's Republic of China (English edition 2000). Vol. 1 Chemical Industry Press, p411, Beijing, China, 2000.

29. Gou YL, Ho AL, Rowlands DK, Chung YW and Chan HC: Effects of Bak Foong Pill on blood coagulation and platelet aggregation. Biol Pharm Bull 26: 241-246, 2003.
30. Rowlands DK, Cui YG, So SC, Tsang LL, Chung YW and Chan HC: Bak Foong Pills induce an analgesic effect by inhibiting nociception via the somatostatin pathway in mice. Cell Biol Int 36: 63-69, 2012.

31. Liu W, Zheng X, Qu Z, et al: Effects of 935-Mhz phone-simulating electromagnetic radiation on endometrial glandular cells during mouse embryo implantation. J Huazhong Univ Sci Technolog Med Sci 32: 755-759, 2012.

32. Rowlands DK, Cui YG, Wong HY, Gou YL and Chan HC: Traditional Chinese medicine Bak Foong Pills alters uterine quiescence-possible role in alleviation of dysmenorrhoeal symptoms. Cell Biol Int 33: 1207-1211, 2009.

33. Morciano A, Romani F, Sagnella F, et al: Assessment of insulin resistance in lean women with polycystic ovary syndrome. Fertil Steril 102: 250-256, 2014

34. Stepto NK, Cassar S, Joham AE, et al: Women with polycystic ovary syndrome have intrinsic insulin resistance on euglycaemic-hyperinsulaemic clamp. Hum Reprod 28: 777-784, 2013.

35. Clirk DA and Dilbaz B: What do we know about metabolic syndrome in adolescents with PCOS? J Turk Ger Gynecol Assoc 15: 49-55, 2014. 